\title{
Creating Shared Value - Grenzen und Vorschläge für eine Weiterentwicklung
}

\section{MARKUS SCHOLZ UND GASTON DE LOS REYES}

Im Creating Shared Value Framework (CSV) werden Maßnahmen vorgeschlagen, mittels derer Unternehmen gleichzeitig gesellschaftliche und ökologische Themen adressieren und Profite generieren können. Doch das CSV-Framework enthält kein Konzept zum Umgang mit konfligierenden ethischen Standards in einer weitgehend globalisierten Wirtschaft. Wenn CSV als ganzheitliches Framework verstanden werden soll, muss es durch ein Norm-Taking und ein Norm-Making Modul ergänzt werden.

Schlagwörter: Creating Shared Value, Management Framework, Norm-Taking, NormMaking

\section{Creating Shared Value - Limits and Suggestions for Further Development}

The Creating Shared V alue Framework (CSV) enables companies to address social and ecological issues and at the same time to generate profits. But the CSV Framework contains no concept for dealing with conflicting ethical standards in a globalized economy. If CSV is to be understood as a holistic framework, it must be supplemented by a norm-taking and a norm-making module.

Keywords: Creating Shared V alue, Management Framework, Norm-Taking, Norm-Making

\section{Einleitung}

Das von Michael Porter und Mark Kramer entwickelte Creating Shared Value Framework (CSV) (vgl. Porter/Kramer 2011) findet in der wissenschaftlichen Gemeinschaft und insbesondere unter UnternehmerInnen und ManagerInnen eine auffallend große Resonanz (vgl. Beschorner 2013; Crane et al. 2014; Driver 2012; FSG 2015; Hartmann/Werhane 2013). Porter und Kramer entwickeln und positionieren CSV als ganzheitliches strategisches Framework, welches gesellschaftliche und

Prof. (FH) Dr. Markus Scholz, Stiftungsprofessur für Corporate Governance \& Business Ethics/FHWien der WKW, Währinger Gürtel 97, A-1180 Wien, Tel.: +43-(1)-476-77-5737, EMail: markus.scholz@fh-wien.ac.at, Forschungsschwerpunkte: Unternehmensstrategie und Unternehmens-ethik; Politische CSR; Theorie-Praxis Verhältnis in den Wirtschaftswissenschaften.

Prof. Gastón de los Reyes, PhD, Assistant Professor of Strategic Management \& Public Policy/ George Washington University School of Business, Funger Hall, Suite 615, 2201 G St. NW, Washington, DC, 20052, USA, Tel.: +1-202-994-5189, E-Mail: gdlr@email.gwu.edu, Forschungsschwerpunkte: Business Ethics; Contracting \& Governance; Strategy \& Corporate Responsibility. 
ökologische Verantwortungen von Unternehmen und das ihnen immanente Profitstreben nicht als Antagonismus auffasst (vgl. Porter/Kramer 2006; 2011; 2014).

Während in der Vergangenheit bereits diverse AutorInnen ähnliche Konzepte vorgelegt haben (vgl. Crane et al. 2014: 134-36; Elkington 1994; 1998; 2001; Emerson 2000; Emerson et al. 2003; Emerson/Bonini 2005; Hart 1997; 2005; London/Hart 2010; Mair/Marti 2006; Moss Kanter 1999; Prahalad/Hart 2002; Vogel 2005), offerieren Porter und Kramer ihre Ideen in einer Art und Weise, welche eine unmittelbare Adaption der vorgeschlagenen Konzepte für PraktikerInnen zulässt. Darüber hinaus greifen sie teilweise auf bekannte und ebenfalls von Michael Porter entwickelte Strategiewerkzeuge zurück, insbesondere auf die Value Chain-Analyse und Porters Diamond-Konzept (vgl. Porter 1998; 2008; Porter/Kramer 2006).

Es steht für uns außer Frage, dass sich das CSV-Framework bereits zu einer festen Größe innerhalb des Spektrums jener Ansätze entwickelt hat, welche die ökologischen und gesellschaftlichen Verantwortungen von Unternehmen adressieren. Gleichzeitig sind wir uns der zum Teil harschen Kritik bewusst, die von führenden UnternehmensethikerInnen gegenüber CSV formuliert wurde (vgl. Beschorner 2013; Crane et al. 2014; Hartman/Werhane 2013). Unser Beitrag greift einige gegenüber CSV geäußerten Kritikpunkte auf, erweitert diese und erläutert mögliche Argumente, welche diesen Vorwürfen eventuell entgegenwirken können.

\section{2. $\quad$ CSV als Management-Framework}

Porter und Kramer positionieren CSV als ganzheitlichen Ansatz, welcher die Rolle und die Aufgaben von Unternehmen in der Gesellschaft neu definiert (vgl. Porter/Kramer 2011; 2014). Wir verstehen CSV deshalb nicht als isolierten Ansatz oder als Add-onStrategiewerkzeug, sondern als Management-Framework. Frameworks, d.h. möglichst ganzheitliche Werkzeuge, waren stets Porters bevorzugte Medien in seinen Beiträgen im Bereich der Unternehmensstrategie. Porter favorisiert diese ganzheitlichen Werkzeuge, da er erkannte, dass isolierte Theoriebausteine und Modelle, wie sie beispielsweise in der Volkswirtschaft gebräuchlich sind, in der anwendungsorientierten Betriebswirtschaftslehre nicht funktionieren: „(We) needed frameworks rather than models (...) ceteris paribus assumptions don't work. Managers must consider everything“" (Argyres/McGahan 2002: 43f.).

Das CSV-Framework kennt zwei miteinander verbundene Ziele, mittels derer ein drittes erreicht werden soll: Porter und Kramer fordern ManagerInnen dazu auf,

1. jene gesellschaftlichen und ökologischen Probleme zu lösen, die mit der Wertschöpfungskette bzw. mit dem Wettbewerbsumfeld des entsprechenden Unternehmens verbunden sind. Gleichzeitig soll diese betriebliche Aktivität nicht um ihrer selbst willen geschehen, sondern ist ein

2. Mittel um Profit zu generieren. Porter und Kramer grenzen sich hier bewusst stark von philanthropischen Ansätzen ab (vgl. Porter/Kramer 2006; 2011; insb. 2014: 149). Gemeinsam sollen diese zwei Bestrebungen zum übergeordneten Ziel 
3. der Re-Legitimierung des kapitalistischen Systems führen (vgl. ebd.). Es ist nicht klar, ob Porter und Kramer neben dem Makroziel (3a: Re-Legitimierung des kapitalistischen Systems), auch ein Mikroziel (3b) erreichen wollen; die individuelle Verbesserung der Legitimität der CSV praktizierenden Unternehmen. Letzteres kann allerdings angenommen werden, da die Autoren ihre Arbeiten für PraktikerInnen aufbereiten und darüber hinaus mit ihrer - eigens für diesen Zweck gegründeten - Non-Profit-Beratungsfirma FSG Unternehmen direkt ansprechen.

Nicht zur Diskussion steht für Porter und Kramer das unbedingte Ziel der Profitorientierung von Unternehmen: „(CSV) is not about 'sharing' the value already created by firms - a redistribution approach" (Porter/Kramer 2011: 65). Dieses bedingungslose Festhalten an der Profitorientierung von Unternehmen mag ein Grund für die Popularität von CSV in der Unternehmenswelt sein. Gleichzeitig steigert diese unbedingte Profitorientierung die kürzlich von diversen prominenten UnternehmensethikerInnen vorgebrachte Kritik an Porters und Kramers Vorschlägen (vgl. Beschorner 2013; Crane et al. 2014; Hartman/Werhane 2013). Diese KritikerInnen zweifeln an der Fähigkeit von CSV mit negativen Externalitäten und sich aufdrängenden Trade-offs umzugehen, welche häufig mit betrieblichen Aktivitäten von Unternehmen einhergehen. Porter und Kramer erwidern auf diese Kritik, dass CSV Unternehmen auffordert, durch ihre betriebliche Tätigkeit verursachte (1) Schäden zu vermeiden und außerdem (2) Gesetze und weitere ethische Standards zu beachten: „Creating shared value presumes compliance with the law and ethical standards, as well as mitigating any harm caused by the business" (Porter/Kramer 2014: 149). Wir betrachten Porters und Kramers normative Forderungen als weitreichend und werden im Folgenden untersuchen, ob und wenn ja, in welcher Art und Weise CSV seinem eigenen Anspruch gerecht wird.

\section{A-Cases, B-Cases und der Umgang mit ethischen Standards}

Wie oben angeführt, positionieren Porter und Kramer CSV als ein ganzheitliches Framework, welches sowohl die Legitimität des Kapitalismus und (in unserer Lesart) auch die Legitimität der darin agierenden Unternehmen wiederherstellen soll. Dennoch begrenzen sie die Reichweite ihres Frameworks explizit, wenn sie einräumen: „NOT ALL societal problems can be solved through shared value solutions“ (Porter/Kramer 2011: 77, Hervorh. im Original; vgl. auch Driver 2012: 421, 426, 429, 431). Porter konkretisiert diese Position an anderer Stelle und verlagert die Verantwortung für die nicht von CSV adressierten Probleme an andere Institutionen: „(I) do not think that all dimensions of all social issues can be addressed by corporations using shared value principles. (...) Maybe someday that will be the case but I think for the foreseeable future, we are going to need a portfolio of institutions in society that are going to be playing different roles" (ebd.: 424).

Wir stimmen mit Porter und Kramer darin überein, dass nicht alle gesellschaftlichen und ökologischen Probleme von Unternehmen gelöst werden können. Ebenso befürworten wir die Vielfalt von gesellschaftlichen Institutionen, welche wiederum diverse Probleme adressieren können. Die Existenz dieser Institutionen entlässt Unternehmen jedoch nicht automatisch aus der Verantwortung, negative 
Auswirkungen, welche aus ihren betrieblichen Aktivitäten resultieren, eigenständig zu vermeiden oder gegebenenfalls zu beheben. Porter und Kramer erkennen dies, wenn sie, wie oben bereits angesprochen, Unternehmen dazu auffordern, neben Gesetzen auch ethische Standards einzuhalten, um entsprechende Schäden zu vermeiden (vgl. Porter/Kramer 2014: 149). Das von Porter und Kramer vorgeschlagene CSVFramework enthält jedoch nicht das nötige Rüstzeug, um ManagerInnen mit entsprechenden Handlungsanweisungen zu versorgen. CSV ist als Framework konzipiert, welches Unternehmen mittels klassischer Strategiewerkzeuge dabei unterstützt A-Cases zu generieren bzw. zu finden. A-Cases sind solche Fälle, in denen die Interessen der Gesellschaft (bzw. der gesellschaftlichen Anspruchsgruppen) mit den Interessen der entsprechenden Unternehmen nicht kollidieren (Win-winSituationen). Beispielsweise unterstützt das Unternehmen Coca-Cola insgesamt fünf Millionen Frauen in Entwicklungsländern mit Mikrokrediten und sorgt zusätzlich für ihre rudimentäre kaufmännische Ausbildung. Als erwartete Folge werden diese Frauen künftig in kioskartigen Kleinstunternehmen diverse Waren, darunter auch Coca-ColaProdukte, ökonomisch nachhaltig anbieten. Coca-Cola generiert hier entlang des Kerngeschäfts (Verkauf von Getränken) einen Shared Value für die Gesellschaft (Stärkung von Frauen; Generierung einer nachhaltigen ökonomischen Infrastruktur) und für das Unternehmen (Aufbau eines leistungsfähigem Vertriebsnetzwerkes) (vgl. Coca-Cola Journey 2013). Porter und Kramer betonen eindrucksvoll die vielfältige Existenz von A-Cases, sowohl in ihren akademischen Publikationen als auch in ihren an die unternehmerische Praxis adressierten Handreichungen, welche sie über ihre Beratungsfirma FSG verbreiten. Die große Attraktivität des CSV-Frameworks liegt u.a. in dem ihm immanenten Mantra der grundsätzlichen Vereinbarkeit von gesellschaftlichen und unternehmerischen Interessen. Es macht somit den Anschein, dass Porter und Kramer ihr Versprechen einlösen können: die Legitimität des Kapitalismus und der darin agierenden Unternehmen wiederherzustellen. Wir werden im Folgenden auf einige Unzulänglichkeiten dieses Ansatzes hinweisen und tentative Lösungsmöglichkeiten zur Behebung dieser Lücken anbieten.

\section{CSV: Keine Hinweise zum Umgang mit B-Cases}

Wir stimmen mit Porter und Kramer dahingehend überein, dass Unternehmen ihr ökologisches und gesellschaftliches Engagement besser als bisher ausrichten können. Der große Verdienst von Porter und Kramer ist die - durch das CSV-Framework systematisierte und in einer für ManagerInnen unmittelbar verständlichen Methodik aufbereitete - Suche nach A-Cases. Das CSV-Framework weist dennoch mindestens zwei (mitunter verknüpfte) Schwachstellen auf: Erstens liefern Porter und Kramer keine Hinweise zum verantwortungsvollen Umgang mit B-Cases. Anders als in den, von Porter und Kramer adressierten, A-Cases verhalten sich unternehmerische und gesellschaftliche Interessen in B-Cases nicht harmonisch, sondern stehen im Konflikt zueinander. Beispielhaft für einen B-Case können die Arbeitsbedingungen in der Textilindustrie in Bangladesch angeführt werden: Der Einsturz der Textilfabrik Rana Plaza im Jahr 2013 kostet 1.138 ArbeiterInnen das Leben. Die Verantwortlichen in der bangladeschischen Legislative und Judikative sind nach diesem Unglück zwar der Empörung und der Wut ihrer MitbürgerInnen ausgesetzt, verbleiben aber gleichzeitig 
weitgehend machtlos gegenüber den Produktionsentscheidungen global agierender Textilunternehmen. Europäische und US-amerikanische Regulierungsbehörden positionieren sich nicht oder nur uneinheitlich hinsichtlich der Zustände der Produktionsstätten in Bangladesch (vgl. Quelch/Rodriguez 2013a; 2013b). In diesem Fall stehen die Profitinteressen der Textilunternehmen und das Bedürfnis der ArbeiterInnen nach sicheren Produktionsbedingungen einander gegenüber. Solange primäre Stakeholdergruppen, wie Shareholder und KonsumentInnen, eine Verbesserung der Produktionsbedingungen nicht konkret einfordern, bedeuten entsprechende betriebliche Maßnahmen Gewinneinbußen für das Unternehmen. Solche Einschnitte in den Profit sind für CSV-Aktivisten nicht hinnehmbar. Porter und Kramer definieren dies eindrücklich, wenn sie betonen, dass CSV kein Umverteilungs- und kein Fair Trade-Ansatz sei (vgl. Porter/Kramer 2011: 65).

\section{CSV: Keine Hinweise zum Umgang mit konfligierenden ethischen Standards}

Das CSV-Framework enthält kein Konzept zum Umgang mit konfligierenden Normen in einer weitgehend globalisierten Unternehmenswelt. Porter und Kramer ebenso wie Milton Friedman $(1962$; 1970) vor ihnen - betonen in ihrem Framework die Relevanz der Einhaltung von ethischen Standards (Porter: ethical standards; Friedman: ethical customs). Keiner dieser Autoren entwickelt einen anwendungsorientierten Ansatz hinsichtlich der Frage, welche ethischen Standards und Konventionen von multinationalen Unternehmen (MNU) zu beachten sind. Diese Thematik ist allerdings zentral für den Bereich der Unternehmensethik. Illustriert werden kann dies am Beispiel von Yahoos Aktivitäten in der Volksrepublik China. Anfang 2002 gab der Internetkonzern u.a. die Nutzerdaten und E-Mails des chinesischen Journalisten Shi Tao an Vertreter der chinesischen Regierung weiter. Als Konsequenz dieser Übermittlung vertraulicher Daten wurde Shi von den chinesischen Behörden verhaftet und ein Jahr später wegen angeblicher „Verbreitung von Staatsgeheimnissen“ zu einer zehnjährigen Gefängnisstrafe verurteilt. Für die Weitergabe der Nutzerdaten und E-Mails wurde Yahoo von MenschenrechtsaktivistInnen und von den US-Behörden scharf kritisiert. Yahoo bestritt die Vorwürfe und argumentierte, dass man vom chinesischen Gesetz her verpflichtet sei, entsprechende Daten an die Behörden weiterzugeben. Gemeinsam mit weiteren prominenten chinesischen DissidentInnen klagte Shis Familie gegen Yahoo. Der Rechtstreit wurde 2007 nach einem Vergleich beigelegt (vgl. Spiegel 2007). Yahoos CEO, Jerry Yang, entschuldigte sich anschließend bei Shis Familie und kündigte an, einen humanitären Fonds für RegimekritikerInnen und deren Angehörige einrichten zu wollen (vgl. ebd.; Brenkert 2009; Dann/Haddow 2008). Wie hätte sich Yahoo hier verhalten sollen? War in diesem Fall der Verweis auf die chinesischen Gesetze ausreichend oder hätte Yahoo die Rechte seiner NutzerInnen auch auf Kosten des eigenen Profits schützen müssen?

Dieser und vergleichbare Fälle können weder von CSV noch von anderen Social Entrepreneurship-Ansätzen adressiert werden, welche gesellschaftliche Probleme durch das bloße Schaffen von innovativen A-Cases lösen möchten. Entsprechende 
Konzepte weisen keinen Mechanismus zur ethischen Reflexion auf, der es ihnen erlauben würde, mit konfligierenden Normen umzugehen.

\section{Mögliche Weiterentwicklungen von CSV: Norm-Taking und Norm- Making Mechanismen}

Wir sind der Ansicht, dass B-Cases in einer weitgehend globalisierten Wirtschaft regelmäßig auftreten und häufig auch vorhersehbar sind. Ein Framework, welches ganz explizit dazu geschaffen wurde, die Legitimität des Kapitalismus und der darin agierenden Unternehmen wiederherzustellen, sollte ManagerInnen mit Handlungsanweisungen beliefern können, wie mit diesen Fällen umzugehen ist. Das CSVFramework kommt dieser Anforderung nicht nach. Wir schlagen deshalb vor, CSV nicht als ganzheitliches Framework zu verstehen, sondern lediglich als Modul eines solchen.

\subsection{ISCT als Norm-Taking Mechanismus}

Um die Defizite von CSV auszugleichen, schlagen wir eine Erweiterung dieses Ansatzes durch klassische Konzepte aus dem Bereich der Unternehmensethik vor. Eine Option für diese Erweiterung ist die von Thomas Dunfee und Thomas Donaldson entwickelte Integrative Social Contracts Theory (ISCT) (vgl. z.B. Donaldson 1996; Donaldson/Dunfee 1999; 2002; Dunfee et al. 1999; Dunfee 2006). Die ISCT überführt die Idee eines Gesellschaftsvertrages (vgl. Rousseau 1762; Rawls 1971) in einen betriebswirtschaftlichen Kontext. ManagerInnen bewegen sich in der Konzeption von ISCT zwischen zwei Ebenen von Normen: hypernorms und microsocial contracts (bzw. microsocial norms). Die übergeordnete Ebene der hypernorms besteht aus global und transkulturell identifizierbaren Normen, welche von den führenden Religionen anerkannt werden sowie den politischen und philosophischen Traditionen entsprechen (vgl. Donaldson/Dunfee 1999: 49-81). Darunter befinden sich microsocial norms, welche auf lokaler Ebene durch die Beteiligung von ökonomischen AkteurInnen definiert werden, z.B. von ArbeitnehmerInnen in einem Unternehmen oder HändlerInnen auf einem Markplatz. Diese Normen werden als authentisch und legitimiert aufgefasst, wenn die beteiligten AkteurInnen die Möglichkeit haben am Normbildungsprozess teilzunehmen, die ökonomische Gemeinschaft (beispielsweise das Unternehmen) zu verlassen und die entsprechende microsocial norm nicht mit einer bypernorm konfligiert. Normen, die diesen Kriterien entsprechen (authentic norms), werden als bindend aufgefasst. Sollten ManagerInnen für ihren Bereich keine bindenden Normen ausmachen können, befinden sie sich, in der Konzeption der ISCT, in einem moral free space. Innerhalb dieses Bereiches verfügen ManagerInnen über einen substanziellen Ermessensspielraum, wie sie mit Stakeholderanforderungen und -interessen umgehen wollen (vgl. z.B. Donaldson 1996; Donaldson/Dunfee 1999; 2002; Dunfee et al. 1999; Dunfee 2006).

Eine Verbindung von ISCT und CSV würde ManagerInnen einen Entscheidungsmechanismus zur Verfügung stellen, wie diese mit konfligierenden ethical standards (Porter) und ethical customs (Friedman) verfahren können: Befinden sich ManagerInnen in einem moral free space wird CSV keinesfalls eingeschränkt. Können hingegen bypernorms und mittels microsocial norms auch authentic norms ausgemacht 
werden, müssen Unternehmen diesen folgen. Wir bezeichnen dieses systematische Auffinden und Abgleichen von Normen in einem unternehmerischen Kontext als Norm-Taking Mechanismus.

\subsection{Unternehmen als politische Akteure: Norm-Making Mechanismen}

Während die ISCT in Bereichen mit einer stark ausgeprägten Normenlandschaft ManagerInnen Auskunft für den Umgang mit B-Cases liefern kann, stößt diese in normativ weniger definierten Bereichen an ihre Grenzen (vgl. Palazzo/Scherer 2007; 2011; Bower et al. 2011). Um das bestehende Regulierungsdefizit für global tätige Unternehmen im Allgemeinen und die anscheinenden Unzulänglichkeiten der ISCT im Besonderen auszugleichen, schlagen wir vor, Unternehmen als Active Corporate Citizens (Citoyens) zu verstehen (vgl. Beschorner/Schank 2012; Scherer/Palazzo 2007): ManagerInnen sollen in diskursive Prozesse mit ihren Stakeholdern eintreten, um in einer wenig ausgeprägten Normenlandschaft neue Normen zu formulieren (vgl. Habermas 1990; Scherer/Palazzo 2007). Unternehmens-vertreterInnen und weitere Stakeholder, die an diesen diskursiven Prozessen teilnehmen, sollten die Vor- und Nachteile ihrer eigenen Positionen berücksichtigen und eine gemeinsame Position entwickeln, welche nicht nur die jeweils eigenen egoistischen, sondern auch die Interessen der anderen Stakeholder hinreichend berücksichtigt (vgl. Palazzo/Scherer 2006; Scherer et al. 2013). Das Ziel dieser Prozesse besteht idealerweise in einer Konsensfindung hinsichtlich neuer Regeln für MNU (im Sinne von microsocial norms), welche in einem nächsten Schritt wiederum über die ISCT ihre Anwendung finden können. Wir bezeichnen diese politische Aktivität von Unternehmen als NormMaking Mechanismus.

\section{Zusammenfassung}

Wir stimmen mit Michael Porter und Mark Kramer dahingehend überein, dass die Legitimität des kapitalistischen Systems sowie die Legitimität der darin agierenden Unternehmen in den vergangenen Jahren massiv abgenommen hat: Unternehmen werden zunehmend als ursächlich für gesellschaftliche, ökologische und ökonomische Krisen betrachtet (vgl. Porter/Kramer 2011: 64). Porter und Kramer präsentieren CSV als ganzheitliches strategisches Framework, welches die Rolle von Unternehmen in der Gesellschaft neu definiert (vgl. Porter/Kramer 2014: 149) und als Antidoton, das die Legitimität des kapitalistischen Systems sowie die Legitimität der darin agierenden Unternehmen wiederherstellen kann. CSV greift viele ältere Ideen aus der CSR-, Sustainability- und Social Entrepreneurship-Forschung auf und präsentiert diese in einer für ManagerInnen unmittelbar umsetzbaren Art und Weise. Die mittlerweile zahlreichen Unternehmen (vgl. FSG 2015), die bereits mit dem CSV-Framework arbeiten und A-Cases generieren, schaffen einen Shared Value. Diese von Porter und Kramer initiierte Bewegung wird zu der von den Autoren avisierten Re-Legitimierung des marktwirtschaftlichen Systems und der Unternehmen beitragen.

Wir verstehen CSV allerdings lediglich als Modul und nicht als ein ganzheitliches Framework. Als solches kann es weder eine umfassende Re-Legitimierung des marktwirtschaftlichen Systems noch der darin agierenden Unternehmen erreichen. CSV verfehlt damit sein eigentliches Hauptziel. Ursächlich dafür ist das dem CSV- 
Framework immanente normative Vakuum im Umgang mit B-Cases, wie auch der Mangel von Mechanismen zum Umgang mit konfligierenden ethischen Standards. CSV enthält ausschließlich Anleitungen zur Generierung von A-Cases. B-Cases, also solche Situationen, in denen gesellschaftliche und unternehmerische Interessen konfligieren, sind allerdings keine Ausnahmeerscheinungen, sondern regelmäßiger Bestandteil betrieblicher Aktivität. Ein Framework, welches die Legitimität von Unternehmen und des wirtschaftlichen Systems wiederherstellen will, sollte diese adressieren können.

Um diese Unzulänglichkeiten auszugleichen, schlagen wir vor, den aktiven CSVAnsatz (Generierung von A-Cases) mit einem Norm-Taking Mechanismus (z.B. ISCT) und einem Norm-Making Mechanismus (Active Corporate Citizenship) zu kombinieren. Ein mit diesen Bestandteilen ausgestattetes Framework kann ManagerInnen dabei unterstützen, A-Cases zu generieren und verantwortungsvoll mit B-Cases umzugehen. Mithilfe eines solchen Frameworks kann einer Re-Legitimierung des wirtschaftlichen Systems und der darin tätigen Unternehmen näher gekommen werden.

Es bleibt das Risiko, dass CSV eine allzu große Begeisterung bei ManagerInnen auslöst und auf der Suche nach Win-win-Cases (A-Cases) deren gesamte Vorstellungskraft einnimmt. Wenn dies geschieht und ManagerInnen als Konsequenz daraus B-Cases und ihre Verantwortung, diese zu adressieren, nicht mehr erkennen dann enthüllt dies eine kaum zu schützende Achillesferse des CSV-Frameworks.

\section{Literaturverzeichnis}

Argyres, N.S./McGahan, A (2002): An Interview with Michael Porter, in: Academy of Management Executive, Vol. 16/No. 2, 43-52.

Beschorner, T. (2013): Creating Shared Value: The One-Trick Pony Approach - A Comment on Michael Porter and Mark Kramer, in: Business Ethics Journal Review, Vol. 1/No. 17, 106-112.

Beschorner, T./Schank, C. (2012): CSR - zur Bürgerrolle und Verantwortung von Unternehmen, in: Schneider, A./Schmidpeter, R. (Hrsg.): Corporate Social Responsibility: Verantwortungsvolle Unternehmensführung in Theorie und Praxis, Berlin: Springer, 155-164.

Bower, J. L./Leonard, H. B./Paine, L. S. (2011): Global Capitalism at Risk. What Are You Doing About It, in: Harvard Business Review, Vol. 89/No. 9, 104-112.

Brenkert, G. G. (2009): Google, Human Rights, and Moral Compromise, in Journal of Business Ethics, Vol. 85/No. 4, 453-478.

Coca-Cola Journey. Redaktion International (2013): Mit starken Frauen zum Erfolg. Studie beweist: Coke ist mit 5by20 auf dem richtigen Weg (17.10.2013). Link: http://www.coca-coladeutschland.de/mit-starken-frauen-zum-erfolg-studie-beweist-coke-ist-mit-5by20-aufdem-richtigen-weg (zuletzt abgerufen am 15.01.2015).

Crane, A./Palazzo, G./Spence, L. J./Matten, D. (2014): Contesting the Value of 'Creating Shared Value', in: California Management Review, Vol. 56/No. 2, 130-153. 
Dann, G. E./Haddow, N. (2008): Just Doing Business or Doing Just Business: Google, Microsoft, Yahoo! and the Business of Censoring China's Internet, in: Journal of Business Ethics, Vol. 79/No. 3, 219-234.

Der Spiegel (2007): Rechtsstreit: Yahoo zahlt chinesische Cyber-Rebellen aus. Link: http://www.spiegel.de/netzwelt/web/rechtsstreit-yahoo-zahlt-chinesische-cyber-re bellen-aus-a-517208.html (zuletzt geprüft am 15.01.2015).

Donaldson, T./Dunfee, T. W. (2002): Ties That Bind in Business Ethics: Social Contracts and Why They Matter, in: Journal of Banking \& Finance, Vol. 26/No. 9, 1853-1865.

Donaldson, T./Dunfee, T. W. (1999): Ties That Bind. A Social Contracts Approach to Business Ethics, Boston, Mass: Harvard Business School Press.

Donaldson, T. (1996): Values in Tension: Ethics away from Home, in: Harvard Business Review, Vol. 74/No. 5, 48-62.

Driver, M. (2012): An Interview with Michael Porter: Social Entrepreneurship and the Transformation of Capitalism, in: Academy of Management Learning \& Education, Vol. 11/No. 3, 421-431.

Dunfee, T. W. (2006): A Critical Perspective of Integrative Social Contracts Theory: Recurring Criticisms and Next Generation Research Topics, in: Journal of Business Ethics, Vol. 68/No. 3, 303-328.

Dunfee, T. W./Smith, N. C./Ross, W. T. (1999): Social Contracts and Marketing Ethics, in: Journal of Marketing, Vol. 63/No. 3, 14-32.

Elkington, J. (2001): The Chrysalis Economy. How Citizen CEOs and Corporations Can Fuse Values and Value Creation, Oxford: Capstone publishing.

Elkington, J. (1998): Cannibals with Forks: The Triple Bottom Line of 21st Century Business, Oxford: Capstone Publishing.

Elkington, J. (1994): Towards the Sustainable Corporation: Win-win-Win Business Strategies for Sustainable Development, in: California Management Review, Vol. 36/No. 2, 90-100.

Emerson, J./Bonini, S. (2005): The Blended Value Map: Tracking the Intersects and Maximizing Blended Value - Building Beyond the Blended Value Map to Sustainable Investing, Philanthropy and Organizations. Link: http://www.blendedvalue.org/wp-content /uploads/2004/02/pdf-max-blendedvalue.pdf (zuletzt geprüft am 06.11.2014).

Emerson, J./Bonini, S./Brehm, K. (2003): The Blended Value Map: Tracking the Intersects and Opportunities of Economic, Social and Enviromental Value Creation. Link: http://www.blendedvalue.org/wp-content/uploads/2004/02/pdf-bv-map.pdf (zuletzt geprüft am 06.11.2014).

Emerson, J. (2000): The Nature of Returns: A Social Capital Markets Inquiry into Elements of Investment and the Blended Value Proposition, Harvard Business School Social Enterprise Series, Working Paper No. 17.

Foundation Strategy Group (FSG) (2015): Full Client List. Link: http://www.fsg.org/Clients/ FullClientList.aspx (zuletzt geprüft am 15.01.2015).

Friedman, M. (1970): The Social Responsibility Of Business Is To Increase Its Profits, in: In New York Times Magazine, 1970 (13. September).

Friedman, M. (1962): Capitalism and Freedom, University of Chicago Press. 
Habermas, J. (1990): Discourse Ethics: Notes on a Program of Philosophical Justification, in J. Habermas (Ed.): Moral Consciousness and Communicative Action, Cambridge, MA: MIT Press, 43-115.

Hart, S. (2005): Capitalism at the Crossroads: The Unlimited Business Opportunities in Solving the World's Difficult Problems, Upper Saddle River,: Wharton School Publishing.

Hart, S. (1997): Beyond Greenings: Strategies for a Sustainable World, in: Harvard Business Review, Vol. 75/No. 1, 66-76.

Hartman, L. P./Werhane, P. H. (2013): Shared Value as an Incomplete Mental Model, in: Business Ethics Journal Review, Vol. 1/No. 6, 36-43.

London, T./Hart, S. (2010): Next-Generation Business Strategies for the Base of the Pyramid: New Approaches for Building Mutual Value, Upper Saddle River: Financial Times Press (NJ).

Mair, J./Marti, I. (2006): Social Entrepreneurship Research: A Source of Explanation, Prediction, and Delight, in: Journal of World Business, Vol. 41/No. 1, 36-44.

Moss Kanter, R. (1999): From Spare Change to Real Change: The Social Sector as Beta Site for Business Innovation, in: Harvard Business Review, Vol. 77/No. 3, 122-128.

Palazzo, G./Scherer, A. G. (2006): Corporate Legitimacy as Deliberation: A Communicative Framework, in: Journal of Business Ethics, Vol. 66/No. 1, 71-88.

Porter, M. E./Kramer, M. R. (2014): A Response to Andrew Crane's et al.'s article, in: California Management Review, Vol. 56/No. 2, 149-151.

Porter, M. E./Kramer, M. R. (2011): Creating Shared Value. How to Reinvent Capitalism - and Unleash a Wave of Innovation and Growth, in: Harvard Business Review, Vol. 89/No. 1, 62-77.

Porter, M. E./Kramer, M. R. (2006): Strategy and Society. The Link Between Competitive Advantage and Corporate Social Responsibility, in: Harvard Business Review, Vol. 84/No. 12, 78-92.

Porter, M. E. (2008): The Five Competitive Forces that Shape Strategy, in: Harvard Business Review, Vol. 86/No. 1, 86-104.

Porter, M. E. (1998): Competitive Strategy, New York, London: Free Press.

Prabalad, C. K./Hart, S. L. (2002): The Fortune at the Bottom of the Pyramid, in: Strategy + Business, Vol. 26/No. 1, 1-14.

Quelch, J. A./Rodriguez, M. L. (2013a): Rana Plaza. Workplace Safety in Bangladesh (A). Harvard Business School Case 514-034, September 2013 (Revised June 2014).

Quelch, J. A./Rodriguez, M. L. (2013b): Rana Plaza. Workplace Safety in Bangladesh (B). Case Harvard Business School Supplement 514-035, September 2013 (Revised June 2014).

Rawls, J. (1971): A Theory of Justice, Harvard University Press.

Rousseau, J.-J. (1762): Du contract social, ou principes du droit politique, Amsterdam: Chez Marc Michel Rey.

Scherer, A. G./Palazzo, G./Seidl, D. (2013): Managing Legitimacy in Complex and Heterogeneous Environments: Sustainable Development in a Globalized World, in: Journal of Management Studies, Vol. 50/No. 2, 259-284.

Scherer, A. G./Palazzo, G. (2011): The New Political Role of Business in a Globalized World: A Review of a New Perspective on CSR and its Implications for the Firm, Governance, and Democracy, in: Journal of Management Studies, Vol. 48/No. 4, 899-931. 
Scherer, A. G./Palazzo, G. (2007): Toward a Political Conception of Corporate Responsibility. Business and Society seen from a Habermasian Perspective, in: Academy Of Management Review, Vol. 32/No. 4, 1096-1120.

Vogel, D. J. (2005): Is there a Market for the Virtue? The Business Case for Corporate Social Responsibility, in: California Management Review, Vol. 47/No. 4, 19-45. 